\title{
Unsolved Problems in Combinatorial Games
}

\author{
RICHARD K. GUY
}

\begin{abstract}
This periodically updated reference resource is intended to put
\end{abstract} eager researchers on the path to fame and (perhaps) fortune.

As in our earlier articles, WW stands for Winning Ways [Berlekamp et al. 1982]. We say that the nim-value of a position is $n$ when its value is the nimber $* n$.

1. Subtraction games are known to be periodic. Investigate the relationship between the subtraction set and the length and structure of the period.

(For subtraction games, see WW, pp. 83-86, 487-498, and Section 4 of [Guy 1996] in this volume, especially the table on page 67 . A move in the subtraction game $S\left(s_{1}, s_{2}, s_{3}, \ldots\right)$ is to take a number of beans from a heap, provided that number is a member of the subtraction set $\left\{s_{1}, s_{2}, s_{3}, \ldots\right\}$. Analysis of such a game and of many other heap games is conveniently recorded by a nim-sequence, $n_{0} n_{1} n_{2} n_{3} \ldots$, meaning that the nim-value of a heap of $h$ beans in this particular game is $n_{h}$ : in symbols, $\mathcal{G}(h)=n_{h}$. Arbitrarily difficult subtraction games can be devised by using infinite subtraction sets: the primes, for example.)

The same question can be asked about partizan subtraction games, in which each player is assigned an individual subtraction set [Fraenkel and Kotzig 1987].

2. Are all finite octal games ultimately periodic? (See WW, pp. 81-115, [Guy 1996, Section 4], p. 67 in this volume. Such games are defined by a code $\mathbf{d}_{0} \cdot \mathbf{d}_{1} \mathbf{d}_{2} \mathbf{d}_{3} \ldots$ If the binary expansion of $\mathbf{d}_{k}$ is $\mathbf{d}_{k}=2^{a_{k}}+2^{b_{k}}+2^{c_{k}}+\cdots$, where $0 \leq a_{k}<b_{k}<c_{k}<\cdots$, then it is legal to remove $k$ beans from a heap, provided that the rest of the heap is left in exactly $a_{k}$ or $b_{k}$ or $c_{k}$ or ... nonempty heaps. Some specimen games are exhibited on page 69.)

Resolve any number of outstanding particular cases, e.g., $\mathbf{6}$ (Officers), $\mathbf{0 6}$, $.14, .36, .64, .74, .76, .004, .005, .006, .007$ (One-dimensional tic-tac-toe,

An earlier version of this collection of problems appeared in Combinatorial Games, Proceedings of Symposia in Applied Mathematics, Vol. 43, 1991. Permission for use courtesy of the American Mathematical Society. We have retained the numbering of problems present in that list. 
Treblecross), $\cdot 016, \cdot 104, \cdot 106, \cdot 114, \cdot 135, \cdot 136, \cdot 142, \cdot 143, \cdot 146, \cdot 162, \cdot 163$, $.172, .324, .336, .342, .362, .371, .374, .404, .414, .416, .444, .454, .564$, $.604, \cdot 606, \cdot 644, \cdot 744, \cdot 764, \cdot 774, \cdot 776$ and Grundy's Game (split a heap into two unequal heaps). Find a game with a period longer than 149459. Explain the structure of the periods of games known to be periodic.

Gangolli and Plambeck [1989] established the ultimate periodicity of four octal games that were previously unknown: $\mathbf{1 6}$ has period 149459 (a prime!), the last exceptional value being $\mathcal{G}(105350)=16$. The game $\cdot \mathbf{5 6}$ has period 144 and last exceptional value $\mathcal{G}(326639)=26$. The games $\cdot \mathbf{1 2 7}$ and $\cdot \mathbf{3 7 6}$ each have period 4 (with cycles of values $4,7,2,1$ and 17, 33, 16, 32 respectively) and last exceptional values $\mathcal{G}(46577)=11$ and $\mathcal{G}(2268247)=42$.

In Problem 38 in Discrete Math. 44 (1983), 331-334, Fraenkel raises questions concerning the computational complexity of octal games. In Problem 39, he and Kotzig define partizan octal games in which distinct octals are assigned to the two players. In Problem 40, Fraenkel introduces poset games, played on a partially ordered set of heaps, each player in turn selecting a heap and removing a positive number of beans from all heaps that are greater or equal to the selected heap in the poset ordering.

3. Examine some hexadecimal games (games with code digits $\mathbf{d}_{k}$ in the interval from $\mathbf{0}$ to $\mathbf{1 5}=\mathbf{F}$ : see WW, pp. 115-116, and the last three examples in the table on page 67 of this book). Obtain conditions for arithmetic periodicity.

Most of the known arithmetically periodic hex games are fairly trivial: $\cdot \mathbf{8}$ is a first cousin of Triplicate Nim, $\cdot \mathbf{A}, \cdot \mathbf{B}, \cdot \mathbf{D}$ and $\cdot \mathbf{F}$ are essentially She-LovesMe-She-Loves-Me-Not, while $\cdot \mathbf{1 A}$ and $\cdot \mathbf{1 B}$, after a couple of exceptional values, display period 2 and saltus 1 . Kenyon's Game, $\mathbf{3 F}$ in the table of page 69 , whose saltus of 3 countered a conjecture of Guy and Smith that it should always be a power of two, is a little more interesting. There must be many others that are possibly accessible to present-day computers.

4. Extend the analysis of Domineering to larger boards. (Left and Right take turns to place dominoes on a checker-board. Left orients her dominoes NorthSouth and Right orients his East-West. Each domino exactly covers two squares of the board and no two dominoes overlap. A player unable to play loses. See [Berlekamp 1988], WW, pp. 495-498, and the articles in this book starting on pages 85 and 311.)

An earlier version of this paper asked what are the values of $4 \times 4$ and $4 \times 5$ boards. David Wolfe has found them to be $\pm\left\{0,\left\{2\left|0,2+_{2} \| 2\right| 0,-{ }_{2}\right\}|| \mid 0\right\}$ and 1 , respectively. Berlekamp has shown that the value of a $4 \times n$ board, if $n$ is odd, is twice that of the corresponding $2 \times n$ board plus a correction term of lower temperature. For the value $g$ of the $4 \times 6$ board, Wolfe's program [1996] needed more than 200 characters to print it. $g$ is incomparable with both $+_{2}$ and $-_{2}$, but he can show that $-4\left(+_{2}\right)<g<3\left(+_{2}\right)$. Dan Calistrate observes that the value of a $6 \times 6$ board is \pm 1 to within 'ish' (infinitesimally shifted). 
Figure 1. Yonghoan Kim's snakes of value $2^{-n}$.

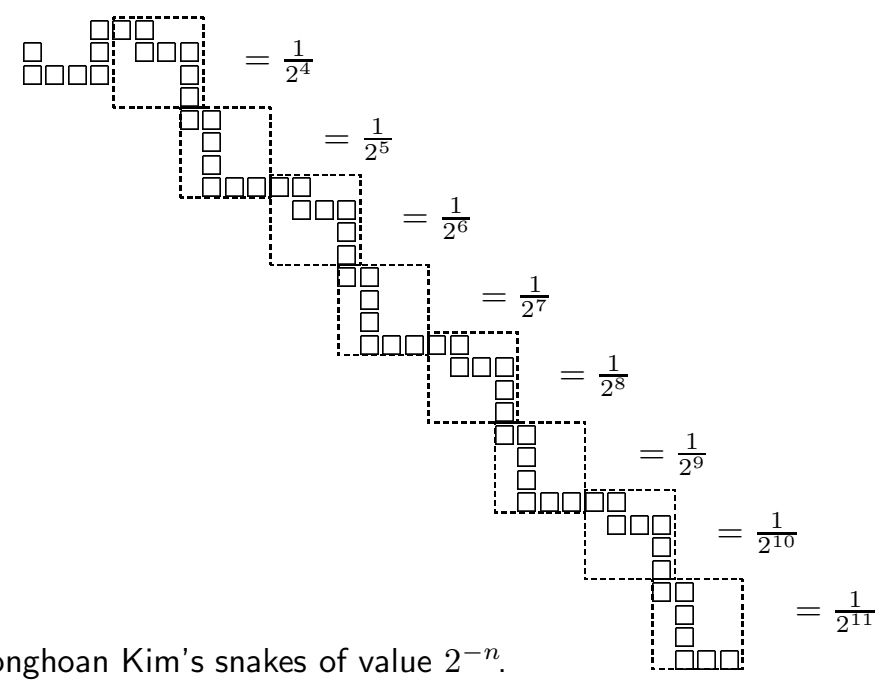

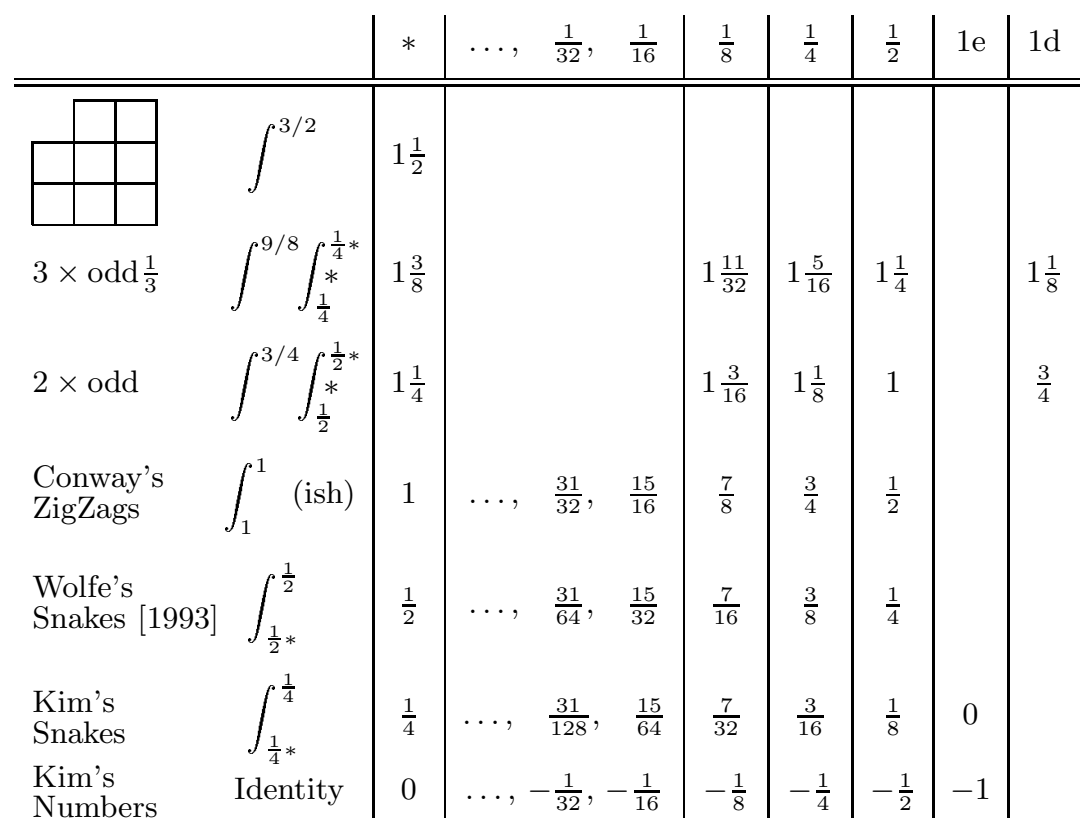

Table 1. Temperatures in Domineering.

Yonghoan Kim [1996] announced that there are snakes of value $2^{-n}$ for all positive integers $n$ (Figure 1). Various subsets of these snakes give a sequence of hot games (the penultimate row of the next table), another sequence of numbers, and several infinitesimals. Wolfe [1993] also has snakes with interesting values.

Table 1 shows temperatures of certain Domineering positions, as reported by Berlekamp. The rows are various overheating and warming operators associated 
with various (families of) positions, and the columns are their arguments. The entries are the temperatures of the resulting games (for references, see Problem 52 below).

Row 2 applies to $3 \times n$ boards with $n$ odd and an additional square appended at one corner; row 3 to $2 \times n$ boards with $n$ odd. The headings $1 \mathrm{e}, 1 \mathrm{~d}$ refer to the argument 1 in the respective cases of even positions, which warm by overheating, and of odd positions, which warm by overheating and then adding $*$, distinguished by a star on the operator sign. If we omit the two entries in the $1 \mathrm{~d}$ column, the rightmost temperature in each row (except the first) matches the leftmost temperature in the next row.

Berlekamp asks, as a hard problem, to characterize all hot Domineering positions to within "ish" (i.e., infinitesimally shifted). As a possibly easier problem he asks for a Domineering position with a new temperature, i.e., one not occurring in the table above.

5. Analyze positions in the game of Go. (Compare [Berlekamp and Wolfe 1994; Berlekamp and Kim 1996; Landman 1996].)

6. In an earlier edition of this paper I asked if Go-Moku (Five-in-a-row, GoBang, Pegotty) is a win for the first player. An affirmative answer for the unrestricted version and for the version where 6-in-a-row doesn't count as a win was given by Allis, van den Herik and Huntjens [1993]. Lustenberger [1967] showed that Four-in-a-row on a $4 \times n$ board is a first-player win for $n$ sufficiently large. Selfridge states that $n=28$ suffices. To find the least $n$ should nowadays be a straightforward computer exercise.

7. Complete the analysis of impartial Eatcakes. (See WW, pp. 269, 271, 276277. Eatcakes is an example of a join or selective compound of games. Each player plays in all the component games. It is played with a number of rectangles, $m_{i} \times n_{i}$; a move is to remove a strip $1 \times n_{i}$ or $m_{i} \times 1$ from each rectangle, either splitting it into two rectangles, or reducing the length or breadth by one. Winner removes the last strip.)

For fixed breadth the remoteness becomes constant when the length is sufficiently large. But 'sufficiently large' seems to be an increasing function of the breadth and doesn't, in the hand calculations already made, settle into any clear pattern. Perhaps computer calculations will reveal something.

8. Complete the analysis of Hotcakes. (See WW, pp. 279-282. Also played with integer-sided rectangles, but as a union or selective compound in which each player moves in some of the components. Left cuts as many rectangles vertically along an integer line as she wishes, and then rotates one from each pair of resulting rectangles through a right angle. Right cuts as many rectangles as he wishes, horizontally into pairs of integer-sided rectangles and rotates one rectangle from each pair through a right angle. The tolls for rectangles with one dimension small are understood, but much remains to be discovered.) 
9. Develop a misère theory for unions of partizan games. (In a union of two or more games, you move in as many component games as you wish. In misère play, the last player loses.)

10. Extend the analysis of Squares Off. (See WW, pp. 299. Played with heaps of beans. A move is to take a perfect square $(>1)$ number of beans from any number of heaps. Heaps of $0,1,2$ or 3 cannot be further reduced. A move leaving a heap of 0 is an overriding win for the player making it. A move leaving 1 is an overriding win for Right, and one leaving 2 is an overriding win for Left. A move leaving 3 doesn't end the game unless all other heaps are of size 3 , in which case the last player wins.)

11. Extend the analysis of Top Entails. (WW, pp. 376-377. Played with stacks of coins. Either split a stack into two smaller ones, or remove the top coin from a stack. In the latter case your opponent's move must use the same stack. Last player wins. Don't leave a stack of 1 on the board, since your opponent must take it and win, since it's now your turn to move in an empty stack!)

Julian West [1996] wrote a program to check a student's work and calculated the first 38,000 values. He found loony positions at 2403 coins, 2505 coins, and 33,243 coins. The authors of WW did not know of a loony stack of more than 3 coins. These results are typical of the apparently quite unpredictable nature of combinatorial games, even when they have quite simple rules.

12. Extend the analysis of All Square. (WW, pp. 385. This game involves complimenting moves after which the same player has an extra bonus move. Note that this happens in Dots-and-Boxes when a box is completed. All Square is played with heaps of beans. A move splits a heap into two smaller ones. If both heap sizes are perfect squares, the player must move again: if he can't he loses!)

13. Analyze the misère version of the octal games of Problem 2. (See [Allemang 1984] and WW, pp. 411-421.)

William L. Sibert made a breakthrough by completing the analysis of misère Kayles; see the postscript to [Sibert and Conway 1992]. Plambeck [1992] has used their method to analyse a few other games, but there's a wide open field here.

14. Moebius, when played on 18 coins has a remarkable pattern. Is there any trace of pattern for larger numbers of coins? Can any estimate be made for the rate of growth of the nim-values?

(Played with a row of coins. A move turns 1, 2, 3, 4 or 5 coins, of which the rightmost must go from heads to tails, to make sure the game satisfies the ending condition. The winner is the player who makes all coins tails. See WW, pp. 432-435; Pless 1991; Curtis 1976; 1977; 1982). 
15. Mogul has an even more striking pattern when played on 24 coins, which has some echoes when played on 40,56 or 64 coins. Thereafter, is there complete chaos? (See Problem 14. A move turns 1, 2, ., 7 coins.)

16. Find an analysis of Antonim with four or more coins. (WW, pp. 459-462. Played with coins on a strip of squares. A move moves a coin from one square to a smaller-numbered square. Only one coin to a square, except that square zero can have any number of coins. It is known that $(a, b, c)$ is a $\mathcal{P}$-position in Antonim just if $(a+1, b+1, c+1)$ is a $\mathcal{P}$-position in Nim, but for more than 3 coins much remains to be discovered.)

17. Extend the analysis of Kotzig's Nim. (WW, pp. 481-483. Players alternately place coins on a circular strip, at most one coin on a square. Each coin must be placed $m$ squares clockwise from the previously placed coin, provided $m$ is in the given move set, and provided the square is not already occupied. The complete analysis is known only for a few small move sets.)

In spite of recent advances [Fraenkel et al. 1995], a great deal remains to be discovered. Is the game eventually periodic in terms of the length of the circle for every finite move set? Analyze the misère version of Kotzig's Nim.

18. Obtain asymptotic estimates for the proportions of $\mathcal{N}-, \mathcal{O}_{-}$and $\mathcal{P}$-positions in Epstein's Put-or-Take-a-Square game. (WW, pp. 484-486. Played with one heap of beans. At each turn there are just two options: to take away or add the largest perfect square number of beans that there is in the heap. Thus 5 is a $\mathcal{P}$-position, because $5 \pm 4$ are both squares; 2 and 3 are $\mathcal{O}$-positions, a win for neither player, since the best play is to go from one to the other, and not to 1 or 4 , which are $\mathcal{N}$-positions.)

19. Simon Norton's game of Tribulations is similar to Epstein's game, but squares are replaced by triangular numbers. Norton conjectures that there are no $\mathcal{O}$-positions, and that the $\mathcal{N}$-positions outnumber the $\mathcal{P}$-positions in golden ratio. True up to 5000 beans.

20. Complete the analysis of D.U.D.E.N.E.Y. (Played with a single heap of beans. Either player may take any number of beans from 1 to $Y$, except that the immediately previous move mustn't be repeated. When you can't move you lose. Analysis easy for $Y$ even, and known for 53/64 of the odd values of $Y$; see WW, pp. 487-489.)

Marc Wallace and Alan Jaffray have made a little progress here, but is the situation one in which there is always a small fraction of cases remaining, no matter how far the analysis is pursued?

21. Schuhstrings is the same as D.U.D.E.N.E.Y., except that a deduction of zero is also allowed, but cannot be immediately repeated (WW, pp. 489-490). 
22. Analyze nonrepeating Nim, in which neither player is allowed to repeat a move. Assume that $b$ beans have been taken from heap $H$, and pick your variant:

medium local: $b$ beans may not be taken from heap $H$ until some other move is made in heap $H$.

short local: $b$ beans may not be taken from heap $H$ on the next move.

long local: $b$ beans may never again be taken from heap $H$.

short global: $b$ beans may not be taken from any heap on the next move.

long global: $b$ beans may never again be taken from any heap.

23. Burning-the-Candle-at-Both-Ends. John Conway and Aviezri Fraenkel ask us to analyze Nim played with a row of heaps. A move may only be made in the leftmost or in the rightmost heap. Of course, when a heap becomes empty, its neighbor becomes the end heap.

Conway has some recollection that the analysis was completed. For one or two heaps the game is the same as Nim. The $\mathcal{P}$-positions for three heaps are $\{n, m, n\}$ with $m \neq n$. If this game is indeed analyzed, then there is also Huband-Spoke Nim, proposed by Fraenkel. One heap is the hub and the others are arranged in rows forming spokes radiating from the hub. There are several versions:

(a) beans may be taken only from a heap at the end of a spoke;

(b) beans may also be taken from the hub;

(c) beans may be taken from the hub only when all the heaps in a spoke are exhausted;

(d) beans may be taken from the hub only when just one spoke remains;

(e) in (b), (c) and (d), when the hub is exhausted, beans may be taken from a heap at either end of any remaining spoke; i.e. the game becomes the sum of a number of games of Burning-the-Candle-at-Both-Ends.

24. Continue the analysis of The Princess and the Roses. (WW, pp. 490494. Played with heaps of beans. Take one bean, or two beans, one from each of two different heaps. The rules seem trivially simple, but the analysis takes on remarkable ramifications.)

25. Analyze the Conway-Paterson game of Sprouts with seven or more spots, or the misère form with five or more spots. (WW, pp. 564-568. A move joins two spots, or a spot to itself by a curve that doesn't meet any other spot or previously drawn curve. When a curve is drawn, a new spot must be placed on it. The valence of any spot must not exceed three. Since this question was asked, Daniel Sleator of AT\&T Bell Laboratories has pushed the normal analysis to about 10 spots and the misère analysis to about 8 .)

26. Extend the analysis of Sylver Coinage. (WW, pp. 575-597. Players alternately name different positive integers, but may not name a number that is 
the sum of previously named ones, with repetitions allowed. Whoever names 1 loses. See [Nowakowski 1991, Section 3].)

27. Extend the analysis of Chomp. (WW, pp. 598-599. Players alternately name divisors of $N$, which may not be multiples of previously named numbers. Whoever names 1 loses.) David Gale offers a prize of US $\$ 100.00$ for the first complete analysis of 3D-Chomp, i.e., where $N$ has three distinct prime divisors, raised to arbitrarily high powers.

28. Extend Uléhla's or Berlekamp's analysis of von Neumann's game from diforests to directed acyclic graphs (WW, pp. 570-572; [Uléhla 1980]). Since Chomp and the superset game [Gale and Neyman 1982] can be described by directed acyclic graphs but not by diforests, the proposed extension could presumably throw some light on these two unsolved games.

29. Prove that Black doesn't have a forced win in Chess.

30. A King and Rook versus King problem. Played on a quarter-infinite board, with initial position WKa1, WRb2 and BKc3. Can White win? If so, in how few moves? It may be better to ask, "what is the smallest board (if any) that White can win on if Black is given a win if he walks off the North or East edges of the board?" Is the answer $9 \times 11$ ? In an earlier edition of this paper I attributed this problem to Simon Norton, but it was proposed as a kriegsspiel problem, with unspecified position of the WK, and with $\mathrm{W}$ to win with probability 1, by Lloyd Shapley around 1960.

31. David Gale's version of Lion and Man. L and M are confined to the nonnegative quadrant of the plane. They move alternately a distance of at most one unit. For which initial positions can L catch M?

Conjecture: L wins if he is northeast of M. This condition is clearly necessary, but the answer is not known even if $\mathrm{M}$ is at the origin and $\mathrm{L}$ is on the diagonal.

Variation. Replace quadrant by wedge-shaped region.

32. Gale's Vingt-et-un. Cards numbered 1 through 10 are laid on the table. $\mathrm{L}$ chooses a card. Then $\mathrm{R}$ chooses cards until his total of chosen cards exceeds the card chosen by L. Then L chooses until her cumulative total exceeds that of $\mathrm{R}$, etc. The first player to get 21 wins. Who is it?

(The rule can be interpreted to mean either "21 exactly" or "21 or more". Jeffery Magnoli, a student of Julian West, thought the second interpretation the more interesting, and found a first-player win in six-card Onze and in eight-card Dix-sept.)

33. Subset Take-away. Given a finite set, players alternately choose proper subsets subject to the rule that, once a subset has been chosen, none of $i t s$ subsets may be chosen later by either player. Last player wins. David Gale conjectures that it's a second-player win; this is true for sets of less than six elements. 
34. Eggleton and Fraenkel ask for a theory of Cannibal Games or an analysis of special families of positions. They are played on an arbitrary finite digraph. Place any numbers of "cannibals" on any vertices. A move is to select a cannibal and move it along a directed edge to a neighboring vertex. If this is occupied, the incoming cannibal eats the whole population (Greedy Cannibals) or just one cannibal (Polite Cannibals). A player unable to move loses. Draws are possible. A partizan version can be played with cannibals of two colors, each eating only the opposite color.

35. Welter's Game on an arbitrary digraph. Place a number of monochromatic tokens on distinct vertices of a directed acyclic graph. A token may be moved to any unoccupied immediate follower. Last player wins. Make a dictionary of $\mathcal{P}$-positions and formulate a winning strategy for other positions. See A. S. Fraenkel and Joseph Kahane, Problem 45, Discrete Math. 45 (1983), 328-329, where a paper is said to be in preparation.

36. Restricted Positrons and Electrons. Fraenkel places a number of Positrons (Pink tokens) and Electrons (Ebony tokens) on distinct vertices of a Welter strip. Any particle can be moved by either player leftward to any square $u$ provided that $u$ is either unoccupied or occupied by a particle of the opposite type. In the latter case, of course, both particles become annihilated (i.e., they are removed from the strip), as physicists tell us positrons and electrons do. Play ends when the excess particles of one type over the other are jammed in the lowest positions of the strip. Last player wins. Formulate a winning strategy for those positions where one exists. Note that if the particles are of one type only, this is Welter's Game; since a strategy is known for Misère Welter (WW, pp. 480-481), it may not be unreasonable to ask for a misère analysis as well. See Problem 47, Discrete Math. 46 (1983), 215-216.

37. General Positrons and Electrons. Like 36, but played on an arbitrary digraph. Last player wins.

38. Fulves's Merger. Karl Fulves, Box 433, Teaneck NJ 07666, sent the following game. Start with heaps of 1, 2, 3, 4, 5, 6 and 7 beans. Two players alternately transfer any number of beans from one heap to another, except that beans may not be transferred from a larger to a smaller heap. The player who makes all the heaps have an even number of beans is the winner.

The total number of beans remains constant, and is even (28 in this case, though one is interested in even numbers in general: a similar game can be played in which the total number is odd and the object is to make all the heaps odd in size). As the moves are nondisjunctive, the Sprague-Grundy theory is not likely to be of help. Fulves's particular game can be solved using Steinhaus's remoteness function [1925] as follows. 
The number of odd heaps is even. If the number of odd heaps is two, there is an immediate win: such a position is an $\mathcal{N}$-position of remoteness 1 . In normal play, $\mathcal{P}$-positions have even remoteness, and $\mathcal{N}$-positions have odd remoteness.

To calculate the remoteness of a position:

- If there are no options, the remoteness is 0 .

- If there is an option of even remoteness, add one to the least such remoteness.

- Otherwise, add one to the greatest odd remoteness.

\section{WIN QUICKLY — LOSE SLOWLY.}

If the number of odd heaps is zero, the position is terminal. All other $\mathcal{P}_{-}$ positions must contain at least four odd heaps. Some examples, with their even remotenesses $r$, are:

\begin{tabular}{|c|c|c|c|c|c|}
\hline P-position & $r$ & $\mathcal{P}$-position & $r$ & $\mathcal{P}$-position & $r$ \\
\hline 11125 & 2 & 1111123 & 4 & 11112418 & 10 \\
\hline 13321 & 4 & 1112221 & 6 & 11113516 & 12 \\
\hline 15517 & 6 & 1113319 & 8 & 11116810 & 18 \\
\hline 17713 & 8 & 1114417 & 10 & 11123812 & 14 \\
\hline 1999 & 10 & 1115515 & 12 & $\begin{array}{llll}11 & 1259 & 9\end{array}$ & 20 \\
\hline \multirow[t]{2}{*}{35911} & 10 & 1116613 & 14 & $\begin{array}{llllll}11 & 1367 & 9\end{array}$ & 22 \\
\hline & & 1117711 & 16 & 11222317 & 10 \\
\hline 112519 & 6 & 111889 & 18 & 11224513 & 14 \\
\hline 113617 & 8 & 1123714 & 12 & 11235511 & 18 \\
\hline 114715 & 10 & 1124911 & 14 & $\begin{array}{lllll}1124 & 2 & 7 & 7\end{array}$ & 20 \\
\hline 123913 & 12 & 1135810 & 18 & 11333413 & 16 \\
\hline 135514 & 12 & 1223515 & 12 & 1134478 & 24 \\
\hline 15679 & 14 & 122779 & 14 & $114445 \quad 9$ & 24 \\
\hline 233317 & 8 & 1233811 & 14 & 12233314 & 16 \\
\hline 255511 & 14 & 125578 & 16 & $123357 \quad 7$ & 22 \\
\hline $2577 \quad 7$ & 14 & 1333315 & 14 & & \\
\hline 334513 & 14 & 1334710 & 16 & & \\
\hline \multirow[t]{5}{*}{33778} & 16 & 133669 & 18 & & \\
\hline & & 1344511 & 18 & & \\
\hline & & 223399 & 16 & & \\
\hline & & 2335510 & 18 & & \\
\hline & & 333568 & 20 & & \\
\hline
\end{tabular}

Table 2 shows a winning strategy from Fulves's starting position.

Richard Nowakowski has investigated some variants of Fulves's game:

1. Take a row of consecutive coins. A move is to take one or more coins from a heap and put them on an adjacent heap of coins provided that the second heap is at least as large as the first. Assume that we start with a row of adjacent coins, that is, each heap consists of one coin. The nim-values are periodic with period seven and the values are 0120310 with no exceptional values. To prove 


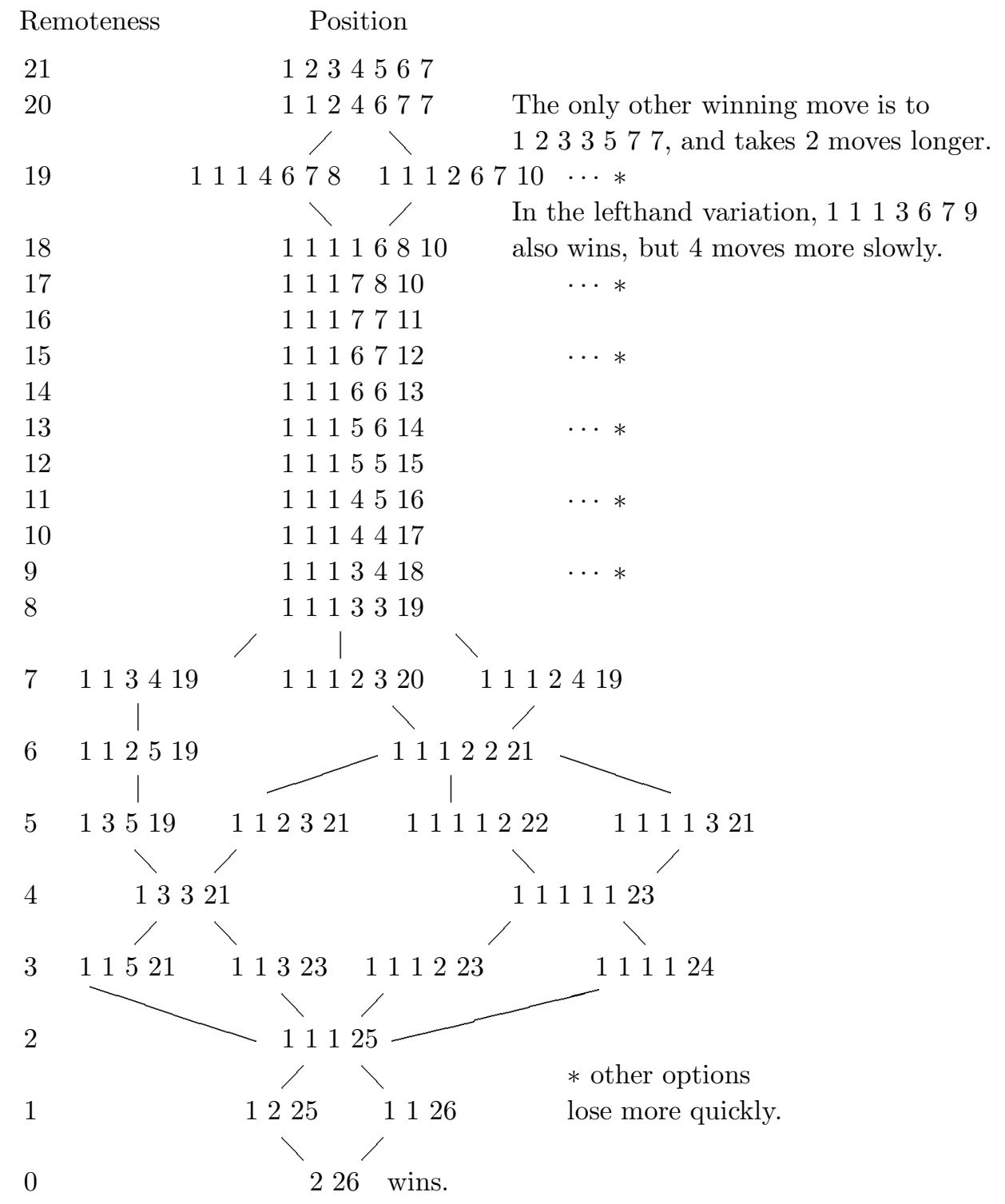

Table 2. Winning strategy for Fulves's game.

this one needs to know the nim-values of the game with the first heap having two coins and also the game with both end heaps haing two coins. The latter is periodic with period seven: the nim-vales are 2100130 with no exceptions. The former also has period seven. The values are 5542564 but they do not start until the game $2.1^{15}$.

The misère version of this variant might be interesting. 
2. Take a row of three consecutive heaps, of sizes $i, j, k$. Then:

(a) If $j>i$ and $j>k$, the nim-value of the position $i . j . k$ is $i \stackrel{*}{+} k$.

(b) If $i=j$ and $j \geq k$, the nim-value is $i+k$.

(c) If either $i>j$ or $k>j$, the nim-value is $j$.

The proof follows easily by consideration of the tables of nim-values for $j=$ $0,1,2,3, \ldots$

39. Sowing or Mancala Games. Kraitchik [1941, p. 282] describes Ruma, which he attributes to Mr. Punga. Bell and Cornelius [1988, pp. 22-38] list Congklak, from Indonesia; Mankal'ah L'ib Al-Ghashim (the game of the unlearned); Leab El-Akil (the game of the wise or intelligent); Wari; Kalah, from Sumeria; Gabata, from Ethiopia; Kiarabu, from Africa; as well as some solitaire games based on a similar idea. Botermans et al. [1989, pp. 174-179] describe Mefuhva from Malawi and Kiuthi from Kenya. Many of these games go back for thousands of years, but several should be susceptible to present day methods of analysis. For a start, see [Erickson 1996] in this volume.

Conway starts with a line of heaps of beans. A typical move is to take (some of) a heap of size $N$ and do something with it that depends on the game and on $N$. He regards the nicest move as what he calls the African move in which all the beans in a heap are picked up and 'sowed' onto successive heaps, and subject to the condition that the last bean must land on a nonempty heap. Beans are sowed to the right if you are Left, to the left if you are Right, or either way if you're playing impartially.

In the partizan version, the position 1 (a single bean) has value 0 , of course; the position $1.1=\{0.2 \mid 2.0\}$ has value $\{0 \mid 0\}=*$; and so does

$$
1.1 .1=\{1.0 .2,2.1 \mid 1.2,2.0 .1\}
$$

since $2.1=\{\mid 3.0\}, 3.0=\{\mid\}=0$ and $1.0 .2=\{\mid 2.1\}$, so that the position 3 has value $0,2.1$ has value $-1,1.0 .2$ value $-2,1.1 .1$ value $\{-2,-1 \mid 1,2\}=0$, 1.1.1.1 value 0 , and 1.1.1.1.1 value $\pm \frac{1}{2}$.

40. Chess again. Noam Elkies [1996] has found endgames with values $0,1, \frac{1}{2}$, $*, \uparrow, \uparrow *, \Uparrow *$, etc. Find endgames with new values. (See also Problems 29, 30 and 45.)

41. Sequential compounds of games have been studied by Stromquist and Ullman [1993]. They mention a more general compound. Let $(P,<)$ be a finite poset and for each $x \in P$ let $G_{x}$ be a game. Consider a game $G(P)$ played as follows. Moves are allowed in any single component $G_{x}$ provided that no legal moves remain in any component $G_{y}$ with $y>x$. A player unable to move loses. The sequential compound is the special case when $(P,<)$ is a chain (or linear order). The sum or disjunctive compound is the case where $(P,<)$ is an antichain. They have no coherent theory of games $G(P)$ for arbitrary posets. 
They list some more specific problems that may be more tractable. Compare Problem 23 above.

42. Beanstalk and Beans-Don't-Talk are games invented by John Isbell and by John Conway [Guy 1986]. Beanstalk is played between Jack and the Giant. The Giant chooses a positive integer, $n_{0}$. Then J. and G. play alternately $n_{1}$, $n_{2}, n_{3}, \ldots$ according to the rule $n_{i+1}=n_{i} / 2$ if $n_{i}$ is even, $=3 n_{i} \pm 1$ if $n_{i}$ is odd; i.e. if $n_{i}$ is even, there's only one option, while if $n_{i}$ is odd there are just two. The winner is the person moving to 1 . If the Giant chooses an odd number $>1$, can Jack always win? Not by using the Greedy Strategy (always descend when it's safe to do so, e.g., play the moves that are underlined in the game below), as may be seen from $n_{0}=7$ (J's moves in parentheses).

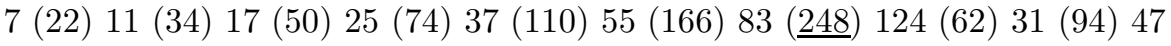
(142 71 (214) 107 (322) 161 (482) 241 (722) 361 (1082) 541 (1624) 812 (406) 203

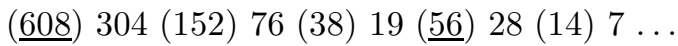

In Beans-Don't-Talk, the move is from $n$ to $(3 n \pm 1) / 2^{*}$ where $2^{*}$ is the highest power of two dividing the numerator; the winner is still the person moving to 1 . Are there any drawn positions? There are certainly drawn plays, e.g., 7 (5) 7 (5) .., but 5 is an $\mathcal{N}$-position because there is the immediate winning option $(5 \times 3+1) / 2^{4}=1$, and 7 is a $\mathcal{P}$-position since the other option $(7 \times 3+1) / 2=11$ is met by $(11 \times 3-1) / 2^{5}=1$. What we want to know is: are there any $\mathcal{O}$-positions (positions of infinite remoteness)?

(For a definition of remoteness see Problem 38 above. There are several unanswered questions about the remotenesses of positions in these two games. Remoteness may also be the best tool we have for Problems 18 and 19 above.)

43. Inverting Hackenbush. John Conway turns Hackenbush (described on page 56 in this volume) into a hot game by amending the move to 'remove an edge of your color and everything thus disconnected from the ground, and then turn the remaining string upside-down and replant it'. The analysis replaces the 'number tree' (WW, p. 25) by a similar tree, but with the smaller binary fractions replaced by increasingly hot games. The game can be generalized to play on trees: a move that prunes the tree at a vertex $V$ includes replanting the tree with $V$ as its root.

44. Konane [Ernst and Berlekamp]. There is much to be discovered about this fascinating and eminently playable game, which exhibits the values $0, *$, $* 2, \uparrow, 2^{-n}$, and many other infinitesimals and also hot values of arbitrarily high temperature.

45. Elwyn Berlekamp asks: 'What is the habitat of $\boldsymbol{* 2}$ ?' This value, defined as $\{0, * \mid 0, *\}$, does not occur in Blockbusting, Hackenbush, Col or Go. It does occur in Konane and $6 \times 6$ Chess. What about Chilled Go, Domineering and $8 \times 8$ Chess? 
46. There are various ways of playing two-dimensional Nim. One form is discussed on p. 313 of WW. Another is proposed by Berman, Fraenkel and Kahane in Problem 41, Discrete Math. 45 (1983), 137-138. Start with a rectangular array of heaps of beans. At each move a row or column is selected and a positive number of beans is taken from some of the heaps in that row or column [Fremlin 1973]. A variant is where beans may be taken only from contiguous heaps. Other variants are played on triangular or hexagonal boards; a special case of this last is Piet Hein's Nimbi, solved by Fraenkel and Herda [1980].

47. Many results are known concerning tiling rectangles with polyominoes. One can extend such problems to disconnected polyominoes. For instance, can a rectangle be tiled by $\square \square \square \square$ ? By $\square \square \square \square \square$ ? If so, what are the smallest rectangles that can be so tiled?

48. Find all words that can be reduced to one peg in one-dimensional Peg Solitaire. (A move is for a peg to jump over an adjacent peg into an empty adjacent space, and remove the jumped-over peg: for instance, $\quad 1101 \rightarrow 0011 \rightarrow 0100$, where 1 represents a peg and 0 an empty space.) Examples of words that can be reduced to one peg are 1, 11, 1101, 110101, 1(10) ${ }^{k} 1$. Georg Gunther, Bert Hartnell and Richard Nowakowski found that for an $n \times 1$ board with one empty space, $n$ must be even and the space must be next but one to the end. If the board is cyclic, the condition is simply $n$ even.

49. Elwyn Berlekamp asks if there is a game that has simple, playable rules, an intricate explicit solution, and is provably NP or harder.

50. John Selfridge asks: is Four-File a draw? Four-File is played on a chessboard with the chess pieces in their usual starting positions, but only on the a-, c-, e- and g-files (a rook, a bishop, a king, a knight and four pawns on each side). The moves are normal chess moves except that play takes place only on these four files; in particular, pawns cannot capture and there is no castling. The aim is to checkmate your opponent's king.

51. Elwyn Berlekamp asks for a complete theory of closed $1 \times n$ Dots-andBoxes, those with starting position

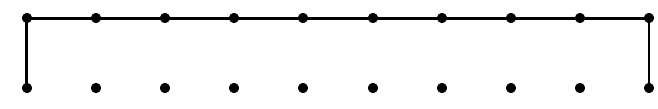

A sample position is

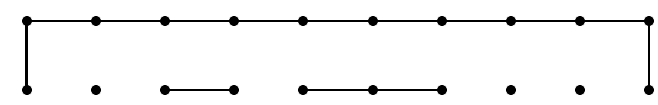

(See WW, Chapter 16.) Are there more nimber decomposition theorems? Compile a datebase of nim-values. 
52. Berlekamp notes that overheating operators provide a very concise way of expressing closed-form solutions to many games, and David Moews observes that monotonicity and linearity depend on the parameters and the domain. How does one play sums of games with varied overheating operators? Find a simple, elegant way of relating the operator parameters to the game. See WW, pp. 163-175, [Berlekamp 1988; Berlekamp and Wolfe 1994; Calistrate 1996].

\section{References}

[Allemang 1984] D. T. Allemang, "Machine Computation with Finite Games", M.Sc. thesis, Cambridge University, 1984.

[Allis et al. 1993] L. V. Allis, H. J. van den Herik, and M. P. H. Huntjens, "Go-Moku solved by new search techniques", pp. 1-9 in Proc. AAAI Fall Symposium on Games: Planning and Learning, AAAI Press Tech. Report FS93-02, Menlo Park, CA.

[Bell and Cornelius 1988] R. Bell and M. Cornelius, Board Games Round the World, Cambridge Univ. Press, Cambridge, 1988.

[Berlekamp 1988] E. R. Berlekamp, "Blockbusting and Domineering", J. Combin. Theory A49 (1988), 67-116.

[Berlekamp and Kim 1996] E. Berlekamp and Y. Kim, "Where is the "thousand-dollar Ko'?", pp. 203-226 in this volume.

[Berlekamp and Wolfe 1994] E. Berlekamp and D. Wolfe, Mathematical Go: Chilling Gets the Last Point, A. K. Peters, Wellesley, MA, 1994. Also published in paperback, with accompanying software, as Mathematical Go: Nightmares for the Professional Go Player, by Ishi Press International, San Jose, CA.

[Berlekamp et al. 1982] E. R. Berlekamp, J. H. Conway, and R. K. Guy, Winning Ways For Your Mathematical Plays, Academic Press, London, 1982.

[Botermans et al. 1989] ack Botermans, Tony Burrett, Pieter van Delft and Carla van Splunteren, The World of Games, Facts on File, New York and Oxford, 1989.

[Calistrate 1996] D. Calistrate, "The reduced canonical form of a game", pp. 409-415 in this volume.

[Curtis 1976] R. T. Curtis, "A new combinatorial approach to $M_{24}$ ", Math. Proc. Cambridge Philos. Soc. 79 (1976), 25-42.

[Curtis 1977] R. T. Curtis, "The maximal subgroups of $M_{24}$ ", Math. Proc. Cambridge Philos. Soc. 81 (1977), 185-192.

[Curtis 1984] R. T. Curtis, "The Steiner system $S(5,6,12)$, the Mathieu group $M_{12}$ and the 'kitten'", pp. 353-358 in Computational Group Theory, Durham, 1982 (edited by M. D. Atkinson), Academic Press, London and New York, 1984).

[Elkies 1996] Noam D. Elkies, "On numbers and endgames", pp. 135-150 in this volume.

[Erickson 1996] J. Erickson, "Sowing Games", pp. 287-297 in this volume.

[Ernst and Berlekamp] Michael D. Ernst and Elwyn Berlekamp, "Playing (Konane) mathematically", to appear in volume of articles in tribute to Martin Gardner (edited by David Klarner), A K Peters, Wellesley, MA.

[Fraenkel and Kotzig 1987] A. S. Fraenkel and A. Kotzig, "Partizan octal games: partizan subtraction games", Internat. J. Game Theory, 16 (1987), 145-154. 
[Fraenkel et al. 1995] A. S. Fraenkel, A. Jaffray, A. Kotzig and G. Sabidussi, "Modular Nim", Theor. Comput. Sci. (Math Games) 143 (1995), 319-333.

[Fremlin 1973] D. Fremlin, "Well-founded games", Eureka 36 (1973), 33-37.

[Gale and Neyman 1982] D. Gale and A. Neyman, "Nim-type games", Internat. J. Game Theory 11 (1982), 17-20.

[Gangolli and Plambeck 1989] A. Gangolli and T. Plambeck, "A note on periodicity in some octal games", Internat. J. Game Theory 18 (1989), 311-320.

[Guy 1986] R. K. Guy, "John Isbell's Game of Beanstalk and John Conway's Game of Beans-Don't-Talk", Math. Mag. 59 (1986), 259-269. (William Jockusch of Carleton College, MN, has corrected the tentative tables on p. 262.)

[Guy 1991] R. K. Guy (editor), Combinatorial Games, Proc. Symp. Appl. Math. 43, Amer. Math. Soc., Providence, RI, 1991.

[Guy 1996] R. K. Guy, "Impartial Games", pp. 61-78 in this volume.

[Kim 1996] Y. Kim, "New values in domineering", Theoret. Comput. Sci. (Math Games) 156 (1996), 263-280.

[Kraitchik 1953] Maurice Kraitchik, Mathematical Recreations, 2nd ed., Dover, New York, 1953.

[Landman 1996] H. A. Landman, "Eyespace Values in Go", pp. 227-257 in this volume.

[Lustenberger 1967] Carlyle Lustenberger, M.S. thesis, Pennsylvania State Univ., 1967.

[Nowakowski 1991] R. J. Nowakowski, “... Welter's Game, Sylver Coinage, Dots-andBoxes, ...", pp. 155-182 in [Guy 1991].

[Plambeck 1992] T. E. Plambeck, "Daisies, Kayles, and the Sibert-Conway decomposition in misère octal games", Theoret. Comput. Sci. (Math Games) 96 (1992), $361-388$.

[Pless 1991] V. Pless, "Games and codes", pp. 101-110 in [Guy 1991].

[Sibert and Conway 1992] W. L. Sibert and J. H. Conway, "Mathematical Kayles" (with a postscript by R. K. Guy), Internat. J. Game Theory 20 (1992), 237-246.

[Steinhaus 1925] H. Steinhaus, "Definicje potrzebne do teorji gry i pościgu", Myśl. Akad. Lwów, 1(1) (1925), 13-14. Reprinted and translated as "Definitions for a theory of games and pursuit", Naval Res. Logist. Quart. 7 (1960), 105-108.

[Stromquist and Ullman 1993] W. Stromquist and D. Ullman, "Sequential Compounds of Combinatorial Games", J. Theoretical Comp. Sci. (Math. Games) 119 (1993), $311-321$.

[Uléhla 1980] J. Uléhla, "A complete analysis of von Neumann's Hackendot", Internat. J. Game Theory 9 (1980), 107-113.

[West 1996] J. West, "New Values for Top Entails", pp. 345-350 in this volume.

[Wolfe 1993] D. Wolfe, "Snakes in Domineering games", Theoret. Comput. Sci. 119 (1993), 323-329.

[Wolfe 1996] D. Wolfe, "The Gamesman's Toolkit", pp. 93-98 in this volume. 
Richard K. GuY

Mathematics and Statistics Department

UNIVERSITY OF CALGARY

2500 University Avenue

Alberta, T2N 1N4

CANAdA 